\title{
Consumo de alimentos ultraprocessados e obesidade abdominal em adolescentes universitários
}

\author{
Ultraprocessed food consumption and with abdominal obesity in university adolescents \\ Consumo de alimentos ultraprocesados y obesidad abdominal en adolescentes \\ universitarios
}

lara Katrynne Fonsêca Oliveira ${ }^{1 *}$, Ednelda de Brito Machado1, Rosana Rodrigues de Sousa1, Adriana de Azevedo Paiva1.

\section{RESUMO}

Objetivo: Avaliar a presença de obesidade abdominal e o consumo de alimentos ultraprocessados (AU) em adolescentes universitários. Métodos: Estudo com abordagem quantitativa, de corte transversal, realizado com adolescentes em Parnaíba-Pl. Ocorreu aplicação de questionários para obtenção de dados socioeconômicos e aplicação do recordatório de $24 \mathrm{~h}$ para obter dados do consumo alimentar. A avaliação antropométrica utilizou os parâmetros: peso, estatura, circunferência da cintura e IMC/Idade. A classificação de AU utilizou o Guia Alimentar para a População Brasileira e os dados foram avaliados pelo software Statistical Package for the Social Sciences (SPSS), versão 21.0. Resultados: 221 adolescentes participaram do estudo e tinham idade média (DP) de 18,3 $\pm 0,7$ anos e $18,2 \pm 0,7$ para o sexo masculino e feminino. $O$ excesso de peso esteve presente em $19 \%$ e $11 \%$ dos meninos e meninas enquanto a obesidade abdominal em $18 \%$ dos adolescentes do sexo masculino e $8 \%$ do sexo feminino. O percentual de contribuição dos AU foi maior na ingestão energética, carboidratos, sódio, gorduras totais e saturada. Conclusão: Adolescentes com obesidade abdominal consumiram mais AU com um maior percentual de contribuição proveniente das gorduras totais e saturadas. É importante que outros estudos sejam realizados para investigar a obesidade abdominal e sua relação com o consumo de AU para favorecer a elaboração de estratégias específicas e eficazes.

Palavras-chave: Adolescente, Obesidade, Circunferência Abdominal, Alimentos Industrializados.

\begin{abstract}
Objective: To evaluate the presence of abdominal obesity and the consumption of ultra-processed foods (AU) in university adolescents. Methods: Cross-sectional quantitative study conducted with adolescents in Parnaíba-PI. Questionnaires were applied to obtain socioeconomic data and the 24-hour recall was used to obtain food consumption data. The anthropometric evaluation used the following parameters: weight, height, waist circumference and BMI / Age. The classification of AU used the Food Guide for the Brazilian Population and the data were evaluated by the Statistical Package for the Social Sciences (SPSS), version 21.0. Results: 221 adolescents participated in the study and had a mean age (SD) of $18,3 \pm 0,7$ years and 18,2 $\pm 0,7$ for males and females. Overweight was present in $19 \%$ and $11 \%$ of boys and girls while abdominal obesity was present in $18 \%$ of male adolescents and $8 \%$ of female adolescents. The percentage of contribution of AU was higher in energy intake, carbohydrates, sodium, total and saturated fat. Conclusion: Adolescents with abdominal obesity consumed more AU with a higher percentage of contribution from total and saturated fat. It is important that other studies be conducted to investigate abdominal obesity and its relationship with UA consumption to favor the development of specific and effective strategies.
\end{abstract}

Key words: Adolescent, Obesity, Abdominal Circumference, Industrialized Foods.

1Universidade Federal do Piauí (UFPI), Teresina-PI. *E-mail: iarakatrynne@hotmail.com 


\section{RESUMEN}

Objetivo: evaluar la presencia de obesidad abdominal y el consumo de alimentos ultraprocesados (AU) en adolescentes universitarios. Métodos: Estudio cuantitativo transversal realizado con adolescentes en Parnaíba-PI. Se aplicaron cuestionarios para obtener datos socioeconómicos y el recuerdo de 24 horas se utilizó para obtener datos de consumo de alimentos. La evaluación antropométrica utilizó los siguientes parámetros: peso, altura, circunferencia de la cintura e IMC / edad. La clasificación de AU utilizó la Guía de Alimentos para la Población Brasileña y los datos fueron evaluados por el Paquete Estadístico para las Ciencias Sociales (SPSS), versión 21.0. Resultados: 221 adolescentes participaron en el estudio y tuvieron una edad media (DE) de $18,3 \pm 0,7$ años y $18,2 \pm 0,7$ para hombres y mujeres. El sobrepeso estuvo presente en el $19 \%$ y el $11 \%$ de los niños y niñas, mientras que la obesidad abdominal estuvo presente en el $18 \%$ de los adolescentes varones y el $8 \%$ de las adolescentes. El porcentaje de contribución de AU fue mayor en la ingesta de energía, carbohidratos, sodio, grasas totales y saturadas. Conclusión: Los adolescentes con obesidad abdominal consumieron más UA con un mayor porcentaje de contribución de las grasas totales y saturadas. Es importante que se realicen otros estudios para investigar la obesidad abdominal y su relación con el consumo de AU para favorecer el desarrollo de estrategias específicas y efectivas

Palabras clave: Adolescente, Obesidad, Circunferencia Abdominal, Alimentos industrializados.

\section{INTRODUÇÃO}

Os produtos ultraprocessados foram definidos com base no Guia Alimentar para a População Brasileira como formulações industriais feitas inteiramente ou majoritariamente por substâncias extraídas de alimentos (óleos, gorduras, açúcar, amido, proteínas), derivadas de constituintes de alimentos ou sintetizadas em laboratórios (corantes, aromatizantes, realçadores de sabor e vários tipos de aditivos usados para conferir aos produtos propriedades sensoriais atraentes) (BRASIL, 2014).

A fabricação de alimentos ultraprocessados são projetados para criar alimentos prontos para consumo, duráveis, acessíveis, convenientes e altamente lucrativos. Positivamente as análises de pesquisas nacionais de compra de alimentos domiciliares e pesquisas dietéticas nacionais realizadas em vários países mostram uma grande variação nas estimativas da contribuição dietética de alimentos ultraprocessados. Estes variaram de $21,5 \%$ da energia total da dieta no Brasil para $57,9 \%$ nos EUA (MONTEIRO CA, et al., 2018). Outros países da Europa e da Ásia relatam tendência similar, indicando que se trata de um cenário global (D'AVILA HF e KIRSTEN VR, 2017).

Segundo Silveira JAC et al. (2017) as práticas alimentares são fatores importantes para a determinação da saúde do indivíduo e quando essa é baseada em alimentos industrializados, ultraprocessados e de baixo valor nutricional que apresentam perfil nutricional obesogênico acarretam danos à saúde e ao bem-estar do indivíduo favorecendo as doenças crônicas não transmissíveis (DCNT) e associada ao aumento das taxas de obesidade como encontrado no Estudo de Riscos Cardiovasculares em Adolescentes (ERICA) de Kuschnir MCC et al. (2016) realizado no Brasil em 2014 que apresentou uma prevalência de 21,3\% de obesidade, com predomínio no sexo masculino.

Diante do contexto alimentar e nutricional em que vivemos, e considerando a perspectiva de potencial malefício da ingestão de alimentos industrializados, o presente estudo tem como objetivo avaliar o estado nutricional, a presença de obesidade abdominal e o consumo de alimentos ultraprocessados em adolescentes universitários.

\section{MÉTODOS}

Estudo transversal realizado com 221 adolescentes de ambos os sexos da Universidade Federal do Piauí (UFPI) no município de Parnaíba-PI. Os estudantes aceitaram voluntariamente participar do estudo, mediante assinatura do Termo de Assentimento e assinatura do Termo de Consentimento Livre e Esclarecido por seus 
responsáveis. Como critérios de elegibilidade considerou-se: ser universitário regularmente matriculado na instituição com idade igual ou inferior a 19 anos e capaz de comunicar-se através da fala. $O$ estudo foi aprovado pelo Comitê de Ética em Pesquisa (CEP/UFPI) com parecer n n. 1394206.

Em relação a avaliação antropométrica foram utilizadas as variáveis: peso, aferido em balança digital da marca Secca ${ }^{\circledR}$; altura, aferida com um antropômetro fixo na parede; cálculo do índice de massa corporal por idade (IMC/ Idade), e; circunferência da cintura (CC), obtida conforme descrito por Scherer R et al. (2013) onde os adolescentes posicionaram-se em pé e utilizou-se uma fita métrica inelástica que circundou a linha natural da cintura, na região mais estreita entre o tórax e o quadril, acima da cicatriz umbilical.

O peso, estatura e CC foram aferidos em duplicatas e posteriormente foi calculada a média. A classificação do estado nutricional segundo o IMC/idade foi realizada de acordo com os critérios propostos pela OMS em 2007 e adotados pelo Ministério da Saúde (BRASIL, 2011) definindo-se: Magreza (< Percentil 3), Eutrofia ( $\geq$ Percentil 3 e $\leq$ Percentil 85), Sobrepeso (> Percentil 85 e < Percentil 97) e Obesidade ( $\geq$ Percentil 97).

A CC foi analisada considerando-se o percentil (P) 90 da própria amostra, sendo esse critério o mais amplamente empregado em estudos que avaliaram a medida de cintura em adolescentes (MAFFEIS C, BANZATO C \& TALAMIN G, 2008; INTERNATIONAL DIABETES FEDERATION, 2007). A presença de obesidade abdominal foi considerada quando os valores eram $>$ Percentil 90 e ausência quando eram $\leq$ Percentil 90.

O consumo alimentar foi investigado por meio do método de Recordatório de 24h (R24h) por ser um método rápido, de baixo custo, exigir pouco esforço dos participantes e não alterar a ingestão de alimentos. A sua aplicação foi baseada nos cinco passos do Multiple Pass Method e replicado em $40 \%$ da amostra. Os alimentos ultraprocessados foram definidos com base no Guia Alimentar para a População Brasileira (MOSHFEGH AJ, et al., 2008).

É importante ressaltar que o diário alimentar não foi utilizado no estudo por ser um tipo de inquérito que dá a possibilidade do indivíduo alterar o consumo por saber que está sendo investigado, além de ser mais oneroso por conta do uso da balança (maior precisão) e ter menor adesão pelos participantes. Contudo é importante lembrar que ambos os métodos estão sujeitos a omissão de informações pelos participantes, porém o R24h por se referir a alimentação do dia anterior impossibilita que ele mude o seu consumo, mais possibilita-o de omitir algum alimento ou refeição no momento da entrevista. Enquanto o diário possibilita que o participante altere sua alimentação para não registrar no formulário da pesquisa.

O consumo total foi analisado usando Programa Virtual Nutri Plus. Em seguida os dados foram exportados para uma planilha do Excel a partir da qual se extraiu as informações referentes aos alimentos ultraprocessados. Tanto para o consumo total quanto para os alimentos ultraprocessados verificou-se: valor calórico, carboidrato, proteína, gordura total, gordura saturada, fibras e sódio. Determinou-se também o percentual de contribuição de energia e nutrientes provenientes dos produtos ultraprocessados em relação ao consumo total.

Os dados do estudo foram avaliados com o auxílio do software Statistical Package for the Social Sciences (SPSS), versão 21.0. Foi considerado valor de significância de 5\% e intervalo de confiança de 95\%. Para análise descritiva utilizou-se frequência simples, média e desvio padrão. A normalidade dos dados foi avaliada usando o teste de Kolmogorov-Smirnov. A partir dos resultados deste teste, decidiu-se quanto ao uso de estatísticas paramétricos ou não-paramétricas. Nas variáveis antropométricas com distribuição normal (peso, altura, IMC, circunferência da cintura) utilizou-se T Student para amostras independentes a fim de comparar as médias no sexo masculino e feminino.

No que concerne ao consumo alimentar, a energia total, a quantidade de carboidrato total e quantidade de carboidratos provenientes dos ultraprocessados apresentaram distribuição normal e tiveram suas médias comparadas segundo o fator com ou sem obesidade abdominal usando $T$ Student para amostras independentes. Comparou-se as médias das demais variáveis de consumo também de acordo com a presença ou não de obesidade usando o teste equivalente não paramétrico: Mann-Whitney.

REAS/EJCH | Vol.11(16) | e1574 | DOI: https://doi.org/10.25248/reas.e1574.2019 Página 3 de 8 


\section{RESULTADOS}

O estudo envolveu 221 adolescentes sendo $42,1 \%$ do sexo masculino (SM) e 57,9\% do sexo feminino (SF) com média de idade e desvio-padrão (DP) de 18,3 \pm 0,7 anos para o SM e 18,2 \pm 0,7 anos para o SF, sem diferença significativa $(p>0,05)$. Quase todos os adolescentes referiram não fumar $(98,9 \%$ dos homens e $99,2 \%$ das mulheres), porém o consumo de álcool foi relatado por $58,1 \%$ dos meninos e $28,9 \%$ das meninas.

Os resultados da avaliação antropométrica dos adolescentes do sexo masculino e feminino estão expostos na (Tabela 1) Nota-se que as médias de peso, altura e circunferência da cintura foram significativamente maiores nos adolescentes do sexo masculino $(p<0,05)$. Entretanto, na avaliação nutricional dos estudantes, não foi encontrada diferenças estatísticas nas médias de IMC por sexo as quais corresponderam no sexo masculino a $22 \pm 3,5 \mathrm{~kg} / \mathrm{m}^{2}$ e no feminino a $21,2 \pm 3,3 \mathrm{~kg} / \mathrm{m}^{2}$, sendo que $85 \%$ das meninas e $72 \%$ dos meninos eram eutróficos (dados não exibidos em tabelas).

Em relação a classificação do estado nutricional pelo IMC/Idade (dados não exibidos em tabela), o excesso de peso (sobrepeso e obesidade) esteve presente em $19 \%$ dos meninos (15\% sobrepeso, $4 \%$ obesidade, $9 \%$ magreza e $72 \%$ eutrofia) e $11 \%$ das meninas ( $9 \%$ sobrepeso, $2 \%$ obesidade, $4 \%$ magreza e $85 \%$ eutrofia). Já a obesidade abdominal esteve presente em $18 \%$ dos adolescentes do sexo masculino e $8 \%$ do sexo feminino (dados não exibidos em tabela).

Tabela 1 - Características antropométricas dos adolescentes universitários de acordo com o sexo. ParnaíbaPI, 2016.

\begin{tabular}{ccccc}
\hline Antropometria & SM Média/DP & SF Média/DP & Teste T & p valor \\
\hline Peso* $^{*}$ & $66,2 \pm 12$ & $55,1 \pm 8,9$ & 7,545 & $<0,01$ \\
Altura* $^{*}$ & $1,73 \pm 5,7$ & $1,62 \pm 5,9$ & 14,743 & $<0,01$ \\
IMC & $22 \pm 3,5$ & $21,2 \pm 3,3$ & 1,881 & 0,06 \\
CC $^{*}$ & $77,3 \pm 8,3$ & $71,6,2 \pm 7,2$ & 5,502 & $<0,01$
\end{tabular}

Fonte: Oliveira IKF, Machado EB, Sousa RR, Paiva AA, 2017. CC - Circunferência da Cintura; DP - Desvio Padrão; IMC - Índice de Massa Corporal; SF - Sexo Feminino; SM - Sexo Masculino * $p<0,05$.

O Quadro 1 apresenta os grupos dos alimentos ultraprocessados e a frequência de consumo desses alimentos, considerando a quantidade de adolescentes que o consumiram pelo menos uma vez ao dia.

Os alimentos ultraprocessados eram ingeridos principalmente em casa ou na lanchonete da Universidade e os grupos que apresentaram maior ingestão foram doces e guloseimas, cereais, gorduras e Fast foods.

Quadro 1 - Alimentos ultraprocessados consumidos pelos adolescentes universitários, segundo o grupo alimentar.

\begin{tabular}{|cc|cc|}
\hline Doces e Guloseimas & $\%$ & Cereais, Gorduras e Fast Food & $\%$ \\
Bolos e Biscoitos & 66,5 & Salgadinhos & 46,1 \\
Balas, Doces e chocolates & 43,4 & Pães & 45,3 \\
Achocolatados em Pó & 20,4 & Margarina & 26,7 \\
Bebidas & $\%$ & Embutidos & $\%$ \\
Refrigerante & 24,9 & Linguiça e Salsicha & 32,1 \\
Bebidas Lácteas e Achocolatados & 19,1 & Bacon e Almôndegas & 19,9 \\
Suco em Pó & 18,1 & Presunto e Defumados & 16,7 \\
\hline
\end{tabular}

Fonte: Oliveira IKF, Machado EB, Sousa RR, Paiva AA, 2017. 
O consumo alimentar total e o consumo de alimentos ultraprocessados com seus respectivos percentuais de contribuição dos alimentos ultraprocessados no consumo total dos universitários do sexo masculino estão apresentados na Tabela 2. Pode-se notar que os homens com ou sem obesidade abdominal tiveram percentuais bem relevantes de contribuição de alimentos ultraprocessados na alimentação principalmente em sódio e gorduras totais e saturadas. Contudo esses percentuais são bem maiores nos indivíduos com obesidade abdominal.

Tabela 2 - Consumo de alimentos ultraprocessados por adolescentes universitários com e sem obesidade abdominal do sexo masculino.

\begin{tabular}{|c|c|c|c|c|c|c|}
\hline & \multicolumn{3}{|c|}{ Com Obesidade Abdominal } & \multicolumn{2}{|c|}{ Sem Obesidade Abdominal } & \multirow[b]{2}{*}{$\% \mathrm{Cl}$} \\
\hline & $\begin{array}{c}\text { CT Médio } \\
\text { (DP) }\end{array}$ & $\begin{array}{l}\text { C. Ultrapr. } \\
\text { (DP) }\end{array}$ & $\% \mathrm{CU}$ & $\begin{array}{r}\text { CT Médio } \\
\text { (DP) }\end{array}$ & $\begin{array}{l}\text { C.Ultrapr. } \\
\text { (DP) }\end{array}$ & \\
\hline \multicolumn{7}{|c|}{ SEXO MASCULINO } \\
\hline Energia $^{1}$ & $\begin{array}{l}2301,9 \\
(852,9)\end{array}$ & $\begin{array}{c}1034,4 \\
(635,3)\end{array}$ & 44,1 & $\begin{array}{c}2459,4 \\
(1283,8)\end{array}$ & $\begin{array}{c}781,8 \\
(573,9)\end{array}$ & 33,9 \\
\hline Proteína² & $\begin{array}{c}95,1 \\
(43,6)\end{array}$ & $\begin{array}{c}29,4 \\
(20,4)\end{array}$ & 34,1 & $\begin{array}{l}113,7 \\
(57,2)\end{array}$ & $\begin{array}{c}22,3 \\
(22,6)\end{array}$ & 24,9 \\
\hline Carboidrato $^{2}$ & $\begin{array}{c}300,2 \\
(109,1)\end{array}$ & $\begin{array}{l}131,7 \\
(73,2)\end{array}$ & 44,3 & $\begin{array}{l}302,7 \\
(166,1)\end{array}$ & $\begin{array}{l}97,5 \\
(73,6)\end{array}$ & 33,3 \\
\hline Fibra $^{2}$ & $\begin{array}{c}18,0 \\
(10,3)\end{array}$ & $\begin{array}{c}5,4 \\
(4,2)\end{array}$ & 32,8 & $\begin{array}{c}18,6 \\
(10,6)\end{array}$ & $\begin{array}{c}3,5 \\
(3,2)\end{array}$ & 24,7 \\
\hline G. Total ${ }^{2}$ & $\begin{array}{c}75,9 \\
(34,3)\end{array}$ & $\begin{array}{c}43,7 \\
(35,8)\end{array}$ & 51,4 & $\begin{array}{c}86,5 \\
(53,2)\end{array}$ & $\begin{array}{c}34,2 \\
(27,2)\end{array}$ & 42,1 \\
\hline G. Saturada ${ }^{2}$ & $\begin{array}{c}27,2 \\
(11,6)\end{array}$ & $\begin{array}{c}14,4 \\
(12,1)\end{array}$ & 48,7 & $\begin{array}{c}31,2 \\
(21,5)\end{array}$ & $\begin{array}{l}11,1 \\
(9,3)\end{array}$ & 38,8 \\
\hline Sódio ${ }^{3}$ & $\begin{array}{c}3228,9 \\
(2074,9)\end{array}$ & $\begin{array}{c}1912,6 \\
(1540,1)\end{array}$ & 59,0 & $\begin{array}{c}3369,7 \\
(2225,5)\end{array}$ & $\begin{array}{c}1487,6 \\
(1438,1)\end{array}$ & 46,1 \\
\hline
\end{tabular}

Fonte: Oliveira IKF, Machado EB, Sousa RR, Paiva AA, 2017. *CT: Consumo Total; ${ }^{*}$ C.Ultrapr.: Consumo de Alimentos Ultraprocessado; *\% CU.: Percentual de Contribuição dos Alimentos Ultraprocessados; *G.: Gordura; 1 Quilocaloria (Kcal); 2- Gamas (g); 3- Miligramas (mg).

A Tabela 3 apresenta os dados do consumo alimentar total e o consumo de alimentos ultraprocessados com seus respectivos percentuais de contribuição para o sexo feminino. As mulheres também apresentaram elevados percentuais de contribuição dos alimentos ultraprocessados na alimentação, exibindo um percentual maior no sódio, gorduras totais e saturadas que são bem mais presentes nos alimentos industrializados.

Tabela 3 - Consumo de alimentos ultraprocessados por adolescentes universitários com e sem obesidade abdominal do sexo feminino.

\begin{tabular}{|c|c|c|c|c|c|c|}
\hline \multirow[b]{3}{*}{ SEXO FEMININ } & \multicolumn{3}{|c|}{ Com Obesidade Abdominal } & \multicolumn{3}{|c|}{ Sem Obesidade Abdominal } \\
\hline & $\begin{array}{l}\text { CT Médio } \\
\text { (DP) }\end{array}$ & $\begin{array}{l}\text { C. Ultrapr. } \\
\text { (DP) }\end{array}$ & $\% \mathrm{CU}$ & $\begin{array}{l}\text { CT Médio } \\
\text { (DP) }\end{array}$ & $\begin{array}{l}\text { C.Ultrapr. } \\
\text { (DP) }\end{array}$ & $\% \mathrm{CU}$ \\
\hline & & & & & & \\
\hline Energia ${ }^{1}$ & $\begin{array}{l}1965,6 \\
(520,0)\end{array}$ & $\begin{array}{c}904,8 \\
(541,6)\end{array}$ & 45,1 & $\begin{array}{l}2153,7 \\
(622,7)\end{array}$ & $\begin{array}{l}927,9 \\
(600)\end{array}$ & 41,5 \\
\hline Proteína ${ }^{2}$ & $\begin{array}{c}82,6 \\
(26,9)\end{array}$ & $\begin{array}{c}23,6 \\
(17,0)\end{array}$ & 33,0 & $\begin{array}{c}90,0 \\
(30,7)\end{array}$ & $\begin{array}{c}26,2 \\
(19,8)\end{array}$ & 31,3 \\
\hline Carboidrato $^{2}$ & $\begin{array}{l}262,2 \\
(87,3)\end{array}$ & $\begin{array}{l}124,4 \\
(81,3)\end{array}$ & 45,9 & $\begin{array}{l}287,2 \\
(85,1)\end{array}$ & $\begin{array}{l}119,0 \\
(73,2)\end{array}$ & 40,4 \\
\hline Fibra $^{2}$ & $\begin{array}{l}16,1 \\
(4,7)\end{array}$ & $\begin{array}{c}5,0 \\
(3,9)\end{array}$ & 33,4 & $\begin{array}{l}19,1 \\
(7,5)\end{array}$ & $\begin{array}{c}4,8 \\
(3,5)\end{array}$ & 27,8 \\
\hline G. Total ${ }^{2}$ & $\begin{array}{c}63 \\
(19,3)\end{array}$ & $\begin{array}{c}35,1 \\
(23,2)\end{array}$ & 51,3 & $\begin{array}{c}71,0 \\
(33,7)\end{array}$ & $\begin{array}{c}38,6 \\
(33,3)\end{array}$ & 50,4 \\
\hline G. Saturada ${ }^{2}$ & $\begin{array}{c}22 \\
(8,2)\end{array}$ & $\begin{array}{l}11,7 \\
(8,2)\end{array}$ & 48,7 & $\begin{array}{c}25,0 \\
(11,5)\end{array}$ & $\begin{array}{c}12,3 \\
(10,7)\end{array}$ & 46,4 \\
\hline Sódio ${ }^{3}$ & $\begin{array}{c}3032 \\
(1810,5)\end{array}$ & $\begin{array}{c}1959,6 \\
(1701,3)\end{array}$ & 64,6 & $\begin{array}{l}2864,5 \\
(1934,1)\end{array}$ & $\begin{array}{l}1592,5 \\
(1342)\end{array}$ & 55,7 \\
\hline
\end{tabular}

Fonte: Oliveira IKF, Machado EB, Sousa RR, Paiva AA, 2017. ${ }^{*}$ CT: Consumo Total; ${ }^{*}$ C.Ultrapr.: Consumo de Alimentos Ultraprocessado; *\% CU.: Percentual de Contribuição dos Alimentos Ultraprocessados; *G.: Gordura; ${ }^{1-}$ Quilocaloria (Kcal); 2- Gamas (g); 3- Miligramas (mg). 
Em relação a comparação das médias de ingestão de energia e nutrientes conforme a presença ou ausência de obesidade abdominal está exposta na Tabela 4.

Observa-se que não houve diferença nas médias de ingestão de energia e nutrientes da alimentação total e da alimentação com ultraprocessados em ambos os gêneros divididos em grupos de acordo com a presença ou ausência de obesidade abdominal, podendo-se perceber de acordo com o presente estudo que apresentam médias de ingestão da alimentação total e dos alimentos ultraprocessados foram semelhantes.

Tabela 4 - Comparação das médias de ingestão de energia e nutrientes segundo a presença de obesidade abdominal.

\begin{tabular}{lccc}
\hline \multicolumn{1}{c}{ Variáveis } & Obesidade abdominal & Sem obesidade abdominal & p valor \\
\hline Energia total & $2185,1 \pm 951,5 \mathrm{Kcal}$ & $2217,6 \pm 727,6 \mathrm{Kcal}$ & $0,865^{\mathrm{a}}$ \\
Energia ultrapr. & $850,2 \pm 105,6 \mathrm{Kcal}$ & $972, \pm 44,3 \mathrm{Kcal}$ & 0,521 \\
Carboidrato total & $280,2 \pm 24,5 \mathrm{~g}$ & $293 \pm 6,9 \mathrm{~g}$ & $0,619^{\mathrm{a}}$ \\
Carboidrato ultrapr. & $112,4 \pm 14,9 \mathrm{~g}$ & $124,3 \pm 5,3 \mathrm{~g}$ & $0,425^{\mathrm{a}}$ \\
Proteína total & $96,4 \pm 8,7 \mathrm{~g}$ & $92,1 \pm 2,6 \mathrm{~g}$ & 0,584 \\
Proteína ultrapr. & $23 \pm 3,7 \mathrm{~g}$ & $27,5 \pm 1,4 \mathrm{~g}$ & 0,232 \\
Gordura total & $73,5 \pm 7,5 \mathrm{~g}$ & $73 \pm 2,4 \mathrm{~g}$ & 0,872 \\
Gordura ultrapr. & $34,7 \pm 4,7 \mathrm{~g}$ & $40,72,5 \mathrm{~g}$ & 0,666 \\
G. Sat. total & $26,1 \pm 3,1 \mathrm{~g}$ & $25,9 \pm 0,8 \mathrm{~g}$ & 0,748 \\
G. Sat. ultrapr. & $11,4 \pm 1,6 \mathrm{~g}$ & $13,2 \pm 0,8 \mathrm{~g}$ & 0,667 \\
Sódio total & $3.182,1 \pm 407,9 \mathrm{mg}$ & $3015,6 \pm 143,7 \mathrm{mg}$ & 0,835 \\
Sódio ultrapr. & $1749,9 \pm 303,7 \mathrm{mg}$ & $1725,2 \pm 103,1 \mathrm{mg}$ & 0,810
\end{tabular}

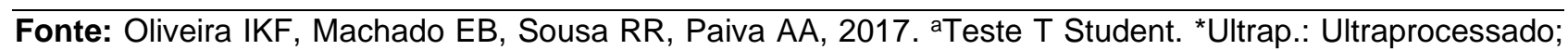
${ }^{*}$ G.Sat.: Gordura Saturada.

\section{DISCUSSÃO}

A utilização dos indicadores antropométricos é recorrente por avaliar o estado nutricional e o risco metabólico e cardiovascular de forma simples e eficaz (BOZZA R, et al., 2009). O principal indicador utilizado para a detecção de obesidade geral é o índice de massa corporal e para obesidade abdominal, a circunferência da cintura, podendo-se associá-los para identificar o risco de complicações metabólicas e utilizando outras variáveis como a circunferência do pescoço, por exemplo (HAUN DR, et al., 1989).

Até o presente momento, não foi estabelecida padronização internacional de pontos de corte para crianças e adolescentes relacionados à CC, porém, muitos estudos fazem essa relação, associar a circunferência da cintura à riscos cardiometabólicos, por conta do risco iminente dessas complicações (PEREIRA PF, et al., 2011).

O excesso de peso segundo o IMC/Idade esteve presente em $19 \%$ dos meninos e $11 \%$ das meninas, estando relacionado ao aumento dos índices de sobrepeso relatado na literatura científica e relacionado como importante preditor de obesidade e desenvolvimento de complicações cardiometabólicas na vida adulta (BARROSO TA, et al., 2017).

Aproximadamente $18 \%$ dos meninos e $8 \%$ das meninas apresentaram obesidade abdominal e os valores de CC elevados têm sido correlacionados com marcadores intermediários de risco cardiometabólicos, tais como hipertensão arterial, dislipidemia, resistência à insulina, síndrome metabólica (SM) entre outros. Além do aumento nos marcadores de disfunção endotelial, como exemplo Proteína-C reativa (PCR) ultrassensível, Fator de Necrose Tumoral Alfa (TNF- $\alpha$ ), entre outros (AVALOSA FC, et al., 2012). 
Kuschnir MCC et al (2016) realizaram um estudo com 37.504 adolescentes brasileiros e encontraram uma prevalência de SM de 2,6\%, sendo essa prevalência 6 vezes maior nos jovens obesos se comparados aos de sobrepeso. Os componentes e fatores de risco para SM que foram encontrados mais frequentes na amostra são CC elevada e High Density Lipoproteins (HDL) baixo que estiveram presente em todos os adolescentes que possuíam a SM e a pressão arterial elevada em alguns jovens. Outros estudos com adolescentes têm-se observado associação significante entre os maus hábitos alimentares e o aumento da CC no desencadeamento de fatores de riscos cardiometabólicos (RINALDI AEM, et al., 2013).

O ambiente universitário acaba dificultando a realização de uma alimentação saudável e balanceada, em virtude da carga de trabalhos acadêmicos, stress, modismos dietéticos e inadequada administração do tempo (LOUREIRO MP, 2016). Segundo Duarte FM et al. (2013) os estudantes acabam preferindo a realização de refeições rápidas, de fácil preparo ou prontas, sem horários definidos e de baixa qualidade nutricional, justificada pela "falta de tempo" que passa a ser bastante frequente.

A partir desse contexto ocorre o aumento no consumo de alimentos ultraprocessados, ricos em gordura, açúcar e sal, e o baixo consumo de legumes, verduras e frutas, associados ao menor gasto energético diário, explicam as tendências crescentes de sobrepeso e obesidade e alterações metabólicas na população infantil e adolescente (SOUZA GS, et al., 2016),

Os alimentos ultraprocessados consumidos pelos adolescentes do presente estudo (quadro 1) assemelhase aos achados de outros estudos onde bolos, biscoitos, salgadinhos, pães, doces, refrescos e refrigerantes também foram os alimentos ultraprocessados mais consumidos pelos adolescentes (BIELEMANN RM, et al., 2015; SOUZA GS, et al., 2016).

O percentual de contribuição energética dos alimentos ultraprocessados na alimentação dos estudantes foi superior ao observado na Pesquisa de Orçamento Familiar na qual esses alimentos contribuíam com $28 \%$ do aporte energético da dieta, mostrando um aumento significativo em pouco mais de uma década (IBGE, 2011; IBGE, 2017).

O presente estudo apontou um percentual maior do que 50\% de gorduras provenientes dos alimentos ultraprocessados em relação ao percentual de gordura total para quase todos os grupos, exceto para o grupo dos homens sem obesidade abdominal, para os quais o consumo foi de $42,1 \%$. O percentual de contribuição das gorduras totais no valor calórico total é de $31,6 \%$. O elevado percentual de gorduras na dieta dos adolescentes também tem sido demonstrado por outros estudos brasileiros (ALBANO RD e SOUZA SB, 2001; GARCIA GCB, et al., 2003).

A Sociedade Brasileira de Cardiologia (2016) recomenda o consumo de até $2000 \mathrm{mg} / \mathrm{dia}$ de sódio ou 5 g/dia de Cloreto de sódio. Em todos os grupos a ingestão diária foi superior a recomendação, tendo um percentual de contribuição bem significativo proveniente dos alimentos ultraprocessados sendo maior que $50 \%$ na maior parte dos grupos. Assumpção D et al. (2012) avaliaram a qualidade global da dieta de adolescentes e a adequação do consumo de cada componente e concluíram que adolescentes com sobrepeso/obesidade apresentaram médias de ingestão superiores de sódio.

De acordo com a tabela 4 não houve diferença estatisticamente significativa nas médias de energia e nutrientes consumidos pelos indivíduos com e sem obesidade abdominal. Vale ressaltar que há uma necessidade de estudos com adolescentes que investiguem mais profundamente esses aspectos, pois são poucos os estudos encontrados que relacionam o consumo de alimentos ultraprocessados em adolescentes de acordo com o sexo e com a presença ou ausência de obesidade abdominal. A utilização de outro tipo de inquérito alimentar como o questionário de frequência de consumo alimentar (QFCA) também seria possível por permitir investigar e avaliar essa relação do consumo de AU e a obesidade a longo prazo.

\section{CONCLUSÃO}

Os adolescentes com obesidade abdominal foram os que mais consumiram alimentos ultraprocessados e o maior percentual de contribuição no consumo total foi proveniente das gorduras totais e saturadas oriundas desses alimentos. Contudo, o crescimento da obesidade abdominal em adolescentes é decorrente de uma 
alimentação baseada em alimentos ultraprocessados que está antecipando a ocorrência de complicações metabólicas e comprometendo a saúde e qualidade de vida desses jovens. É importante salientar que outros estudos sejam realizados para investigar a obesidade abdominal e sua relação com o consumo de alimentos ultraprocessados, espera-se que os resultados do estudo ajudem no controle da obesidade e na elaboração de estratégias específicas e eficazes.

\section{REFERÊNCIAS}

1. ALBANO RD, SOUZA SB. Estado nutricional de adolescentes: "risco de sobrepeso" e "sobrepeso" em uma escola pública do Município de São Paulo. Cad. Saúde Pública [online], 2001; 17(1):941-947.

2. ASSUMPÇÃO D, BARROS MBA, FISBERG RM et al. Qualidade da dieta de adolescentes: estudo de base populacional em Campinas. Revista Brasileira de Epidemiologia, 2012; 15(3):605-616.

3. AVALOSA C, DIAZ C, MARTINEZ A et al. Waist circumference percentiles in children and adolescentes between 6 and 14 years from Santiago, Chile. Endocrinologia e Nutrição, 2012; 59(5):296-303.

4. BARROSO AT, MARINS LB, ALVES R et al. Association of Central Obesity with the Incidence of Cardiovascular Diseases and Risk Factors. International Journal of Cardiovascular Sciences, 2017; 30(5):416-424.

5. BIELEMANN RM, MOTTA JVS, MINTEN GC et al. Consumo de alimentos ultraprocessados e impacto na dieta de adultos jovens. Revista de Saúde Pública, 2015; 49(1):1-10.

6. BOZZA R, NETO AS, ULBRICH AZ et al. Circunferência da cintura, índice de massa corporal e fatores de risco cardiovascular na adolescência. Rev Bras Cineantropom Desempenho Hum., 2009; 11(3):286-291.

7. BRASIL, Ministério da Saúde. Guia alimentar para a população brasileira. Brasília: Ministério da Saúde, 2014.

8. BRASIL, Ministério da Saúde. Orientações para a coleta e análise de dados antropométricos em serviços de saúde: Norma Técnica do Sistema de Vigilância Alimentar e Nutricional - SISVAN. Brasília: Ministério da Saúde, 2011.

9. D'AVILA HF, KIRSTEN VR. Energy intake from ultra-processed foods among adolescents. Rev Paul Pediatr., 2017; 35(1):54-60.

10. Duarte FM, ALMEIDA SDS, MARTINS KA. Alimentação fora do domicílio de universitários de alguns cursos da área da saúde de uma instituição privada. Mundo da Saúde, 2013; 37(3):288-298.

11. GARCIA GCB, GAMBARDELLA AMD, FRUTUOSO MFP. Estado nutricional e consumo alimentar de adolescentes de um centro de juventude da cidade de São Paulo. Rev. Nutr. [online], 2003; 16(1):41-50.

12. HAUN DR, PITANGA FJG, LESSA I. Razão cintura/estatura comparado a outros indicadores antropométricos de obesidade como preditor de risco coronariano elevado. Rev Assoc Med Bras., 2009; 55(6):705-711.

13. IBGE- Instituto Brasileiro de Geografia Estatística. Rendimento domiciliar per capita 2016. Rio de Janeiro. 2017.

14. IBGE- Instituto Brasileiro de Geografia e Estatística. Pesquisa de orçamentos familiares 2008-2009: análise do consumo alimentar pessoal no Brasil. Rio de Janeiro. 2011.

15. IDF- International Diabetes Federation. The IDF consensus definition of the Metabolic Syndrome in children and adolescents. 2007.

16. KUSCHNIR MCC, BLOCH KV, SZKLO M et al. ERICA: Prevalência de Síndrome Metabólica em Adolescentes Brasileiros. Revista de Saúde Pública, 2016; 1(1):1-13.

17. LOUREIRO MP. Estado nutricional e hábitos alimentares de universitários. Segurança Alimentar e Nutricional, 2016; 23(2):955-972.

18. MAFFEIS C, BANZATO C, TALAMINI L et al. Obesity Study Group of the Italian Society of Pediatric Endocrinology and Diabetology. Waist-to-height ratio, a useful index to identify high metabolic risk in overweight children. Jornal de Pediatria, 2008; 152(2):207-213.

19. MONTEIRO CA, MOUBARAC JC, LEVY RB et al. Household availability of ultra-processed foods and obesity in nineteen European countries. Public Health Nutr., 2018; 21(1):18-26.

20. MOSHFEGH AJ, RODES DG, BAER DJ et al. The US Department of Agriculture Automated Multiple-Pass Method reduces bias in the collection of energy intakes. Am J Clin Nutr., 2008; 88(2):324-332.

21. PEREIRA PF, SERRANO HMS, CARVALHO GQ et al. Circunferência da cintura e relação cintura/estatura: úteis para identificar risco metabólico em adolescentes do sexo feminino? Rev Paul Pediatr., 2011; 29(3):372-377.

22. RINALDI AEM, NOGUEIRA PCK, RIYUZO MC et al. Prevalência de pressão arterial elevada em crianças e adolescentes do ensino fundamental. Revista Paulista de Pediatria, 2013; 30(1):79-86.

23. SANTOS JS, COSTA MCO, SOBRINHO CLN et al. Perfil antropométrico e consumo alimentar de adolescentes de Teixeira de Freitas - Bahia. Revista de Nutrição, 2005; 18(5):623-632.

24. SCHERER R, SCHERER F, CONDE SR et al. Nutritional status and prevalence of chronic diseases among elderly in the countryside of Rio Grande do Sul state, Brazil. Rev. bras. geriatr. gerontol., 2013; 16(4):769-779.

25. SILVEIRA JAC, MENESES SS, QUINTANA PT et al. Associação entre o excesso de peso e o consumo de alimentos ultraprocessados e bebidas açucaradas por diferentes grupos vegetarianos. Rev. Nutr.[online]., 2017; 30(4):431-44.

26. SBC- Sociedade Brasileira de Cardiologia. The Brazilian Guideline of Arterial Hypertension. 2016; 107(3):8-10.

27. SOUZA GS, QUADROS TMB, GORDIA AP et al. Revisão da literatura sobre extremos antropométricos em crianças e adolescentes: Prevalência, riscos à saúde e fatores sociodemográficos associados. Revista de Atenção à Saúde, 2015; 13(45):102-113.

28. SOUZA AM, BARUFALDI LA, ABREU GA et al. ERICA: ingestão de macro e micronutrientes em adolescentes brasileiros. Rev Saúde Pública. 2016; 50(1):1-15. 